\title{
Features of Polymethacrylic Acid and Poly-2-Methyl-5-Vinylpyridine Hydrogels Remote Interaction in an Aqueous Medium
}

\author{
Alimbekova Bagyla Talgatovna, Korganbayeva Zhanar Kozhamberdikyzy, Himersen Huangul, Kondaurov \\ Ruslan Gennadievich and Jumadilov Talkybek Kozhataevich* \\ JSC “Institute of Chemical Sciences Named after A. B. Bekturov”, Kazakh National Pedagogical University after Abai, Almaty \\ 050010, Republic of Kazakhstan
}

Received: January 28, 2014 / Accepted: February 16, 2014 / Published: March 25, 2014.

\begin{abstract}
The remote interaction of polymethacrylic acid hydrogel with a poly-2-methyl-5-vinylpyridine hydrogel was studied. The aim of work was to study the dependence of the swelling coefficient, the conductivity and the $\mathrm{pH}$ of the water solutions of intergel system at different mass ratios from time were studied. The goal was achieved by using following methods: $\mathrm{pH}$-metry, conductometry and gravimetry.
\end{abstract}

Key words: Polyacrylic acid, poly-2-methl-5-vinylpyridine, intergel system.

\section{Introduction}

Earlier in the systematic study of hydrogels complexing properties the problem arose on the two hydrogels interaction study. Due to macroscopic size and mobility dispersion of hydrogels it is impossible to study these substances by traditional way [1]. Therefore, it was tasked to study the possibility of interaction of polymer networks at a distance in the absence of direct contact between them. Subsequent works showed that intergel system consisting of two hydrogels exhibit selectivity for metal ions [2-5]. It was assumed that during remote interaction interacting hydrogels transform into more active state as a result of mutual activation [6,7]. In order to clarify the possibility of remote interaction by using following methods: conductometry, pH-metry, and gravimetry electrochemical properties of PMAA (polymethacrylic acid) hydrogel and P2M5VP

\footnotetext{
"Corresponding author: Jumadilov Talkybek Kozhataevich, Professor, research fields: macromolecular compounds and remote interaction of hydrogels. E-mail: jumadilov@mail.ru.
}

(poly-2-methyl-5-vinylpyridine) hydrogel intergel system aqueous solutions and changes of swelling ratio in remote interactions were studied.

\section{Experiments}

\subsection{Equipments}

For measurement of electroconductivity, conductometer "MARK 603" (Russia) was used, pH of solutions was measured on $\mathrm{pH}$ meter "Seven Easy" (METTLER TOLEDO, China). Swelling coefficient $K_{s}$ was defined by weighting of hydrogel swollen samples on electronic scales "SHIMADZU AY220" (Japan).

\subsection{Materials}

Studies were carried out in distillated water medium. Polymethacrylic acid hydrogels were synthesized in the presence of crosslinking agent $\mathrm{N}, \mathrm{N}$-methylene-bis-acrylamide and redox system $\mathrm{K}_{2} \mathrm{~S}_{2} \mathrm{O}_{8}, \quad \mathrm{Na}_{2} \mathrm{~S}_{2} \mathrm{O}_{3}$. Poly-2-methyl-5-vinylpyridine hydrogel was synthesized by crosslinking of a linear 
poly-2-methyl-5-vinylpyridine with epichlorohydrin in a medium of dimethylformamide.

\subsection{Methods}

For intergel pair drawing up poly-2-methyl5-vinylpyridine was used. Swelling coefficients of hydrogels were $K_{s w}($ gPMAA $)=20.65, K_{s w}($ gP2M5VP) $=3.20$. Experiments were carried out at a room temperature. Study of intergel systems was made by this way: Each hydrogel was located in separated glass weighing bottle pores of which are permeable for low-molecular ions and molecules, but it is not permeable for a dispersion hydrogels.

Then weighing bottles with hydrogels were located in glasses with distilled water. Electroconductivity and $\mathrm{pH}$ of overgel liquid was measured by taking out of weighing bottles with hydrogels from the glass. Swelling coefficient was calculated as the difference of weights of weighing bottle with hydrogel and empty weighing bottle according to Eq. (1).

$$
K_{s w}=\frac{m_{2}-m_{1}}{m_{1}}
$$

where, $m_{1}$ - weight of dry hydrogel, $m_{2}$ - weight of swollen hydrogel.

\section{Results and Discussion}

Obtained electrical conductivity dependencies of intergel system's various ratios from time are shown in Fig. 1. Fig. 1 shows that electrical conductivity increases with time for all ratios of gPMAA:gP2M5VP. However, the behavior of electrical conductivity for different ratios differs. With increasing contact time with aqueous medium areas of electrical conductivity maximum and minimum observed. At $60 \mathrm{~min}$, minimum becomes noticeable at ratios $5: 1$ and $3: 3$. At the ratios $4: 2$ and $2: 4$, the authors observed the appearance of the maximum electrical conductivity area.

The minimum electrical conductivity of intergel system caused by binding cleaved from the carboxyl group proton by heteroatom of vinylpyridine's nitrogen. At the moment of immersion in aqueous medium, both hydrogels begin to swell as a result of interactions with water molecules. Carbonyl groups are at first ionized and then dissociate at the carboxylate anion- $\mathrm{COO}^{-}$and hydrogen ions $\mathrm{H}^{+}$. Thus, in the process of dissociation in aqueous medium proton ions appear. Cationic hydrogel of poly-2-methyl-5-vinylpyridine is ionized in aqueous

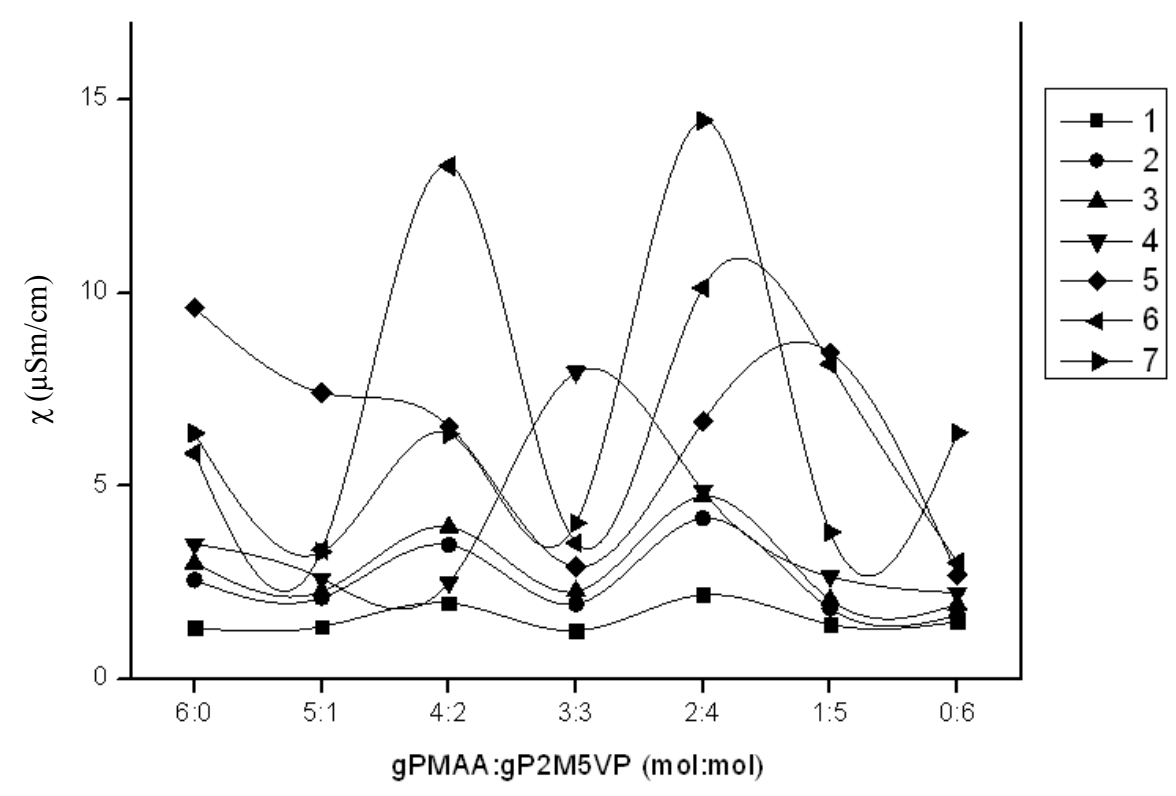

Fig. 1 Dependencies of polymethacrylic acid hydrogel-poly-2-methyl-5-vinylpyridine hydrogel intergel system's specific electrical conductivity from time in an aqueous medium.

Curves' description: $1-20 \mathrm{~min}$; 2-40 min; 3-60 min; 4-120 min; 5-240 min; 6-480 $\mathrm{min}$; 7-1,440 min. 
medium under acceding hydrogen ions formed by dissociation of carboxyl groups and water molecules on ions $\mathrm{H}^{+}, \mathrm{OH}^{-}$. In these interactions, the total content of positive charges in an aqueous medium decreases. Due to the bulky sizes hydrogels with charged ions have no mobility in an aqueous medium.

Fig. 2 shows changes in the hydrogen ions concentration in an aqueous medium in the presence of intergel system PMAA gel and P2M5VP gel. Obtained results show that $\mathrm{H}^{+}$cleavage rate in the initial moment during dissociation (Fig. 2-curves 1, $2,3)$ is higher than $\mathrm{H}^{+}$binding rate with polybasis. With decreasing of swelling rate of $-\mathrm{COOH}$ groups dissociation occurs, thereby $\mathrm{pH}$ of medium increases at ratio 5:1. So, concentration of $\mathrm{H}^{+}$ions determines equilibrium of two processes: (1) swelling rate, at which $\mathrm{H}^{+}$ions are allocated in the solution as a result of dissociation of - $\mathrm{COOH}$ groups; (2) binding rate of $\mathrm{H}^{+}$ions with links of polybasis. With the increasing share of poly-2-methl-5-vinylpyridine $\mathrm{pH}$ of solution gradually falls, passing through a minimum at a ratio gPMAA:gP2M5VP = 4:2. Low hydrogen concentrations in an aqueous medium for intergel system are observed at ratios of gPMAA:gP2M5VP = $5: 1$ and $3: 1$, which practically coincide with the results of the electrical conductivity.

Fig. 3 shows that as a result of polymeric hydrogels long-range effect polyacid swelling coefficient $\left(K_{s w}\right)$ change is observed. On the dependencies $K_{s w}$-ratio gPMAA:gP2M5VP, we observe a significant increase in the polyacid swelling at ratio of gPMAA:gP2M5VP $=4: 2$. These results indicate that at this ratio along the internodal links of polyacid maximum density of charged groups occurs, due to the high ionization of the acid groups leading to spreading out of the acid hydrogel polymer chains. Swelling coefficient increase is also observed at ratio of gPMAA:gP2M5VP = 2:4.

Fig. 4 characterizes swelling coefficient change of the basic hydrogel poly-2-methyl-5-vinylpyridine in an aqueous medium in the presence of polymethacrylic acid hydrogel. With the polyacid content increase gP2M5VP additional swelling is observed. This indicates additional polibasis links activation. Due to the proximity of curves maximum and minimum points are difficult to detect. General appearance of the curves indicates an increase in swelling coefficient of cationic hydrogel in presence of acid hydrogel.

As is known, poly-2-methyl-5-vinylpyridine gel slightly swells in an aqueous medium due to it is weak poliobasis. Swelling coefficient of gP2M5VP increase by 7.8 times indicates a strong degree of vinylpyridine's ionization. This may be due only to

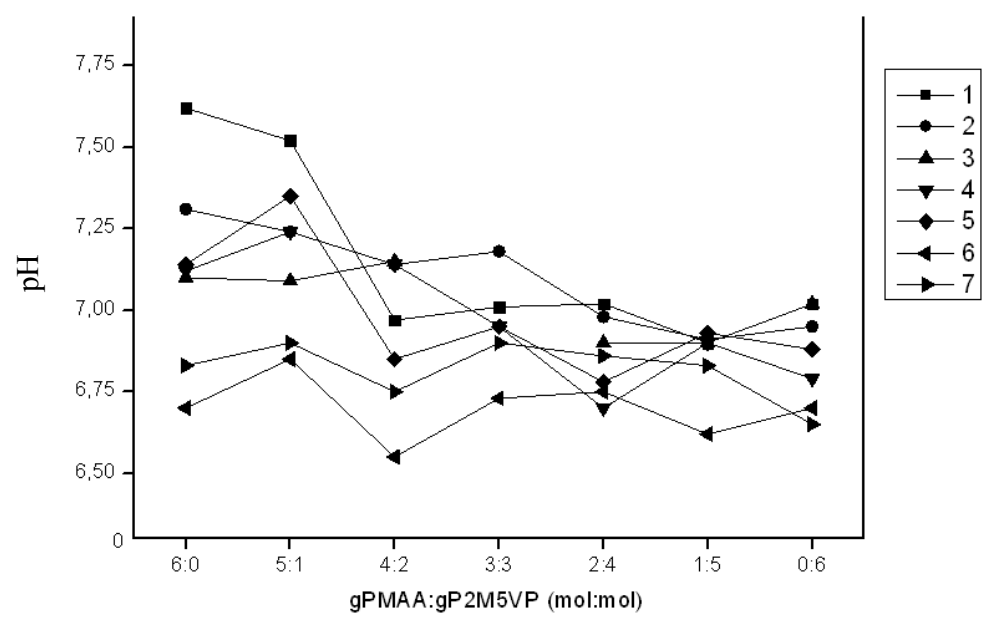

Fig. 2 Dependencies of polymethacrylic acid hydrogel-poly-2-methyl-5-vinylpyridine hydrogel intergel system solution's pH from time.

Curves' description: 1-20 min; 2-40 min; 3-60 min; 4-120 min; 5-240 min; 6-480 min; 7-1,440 min. 


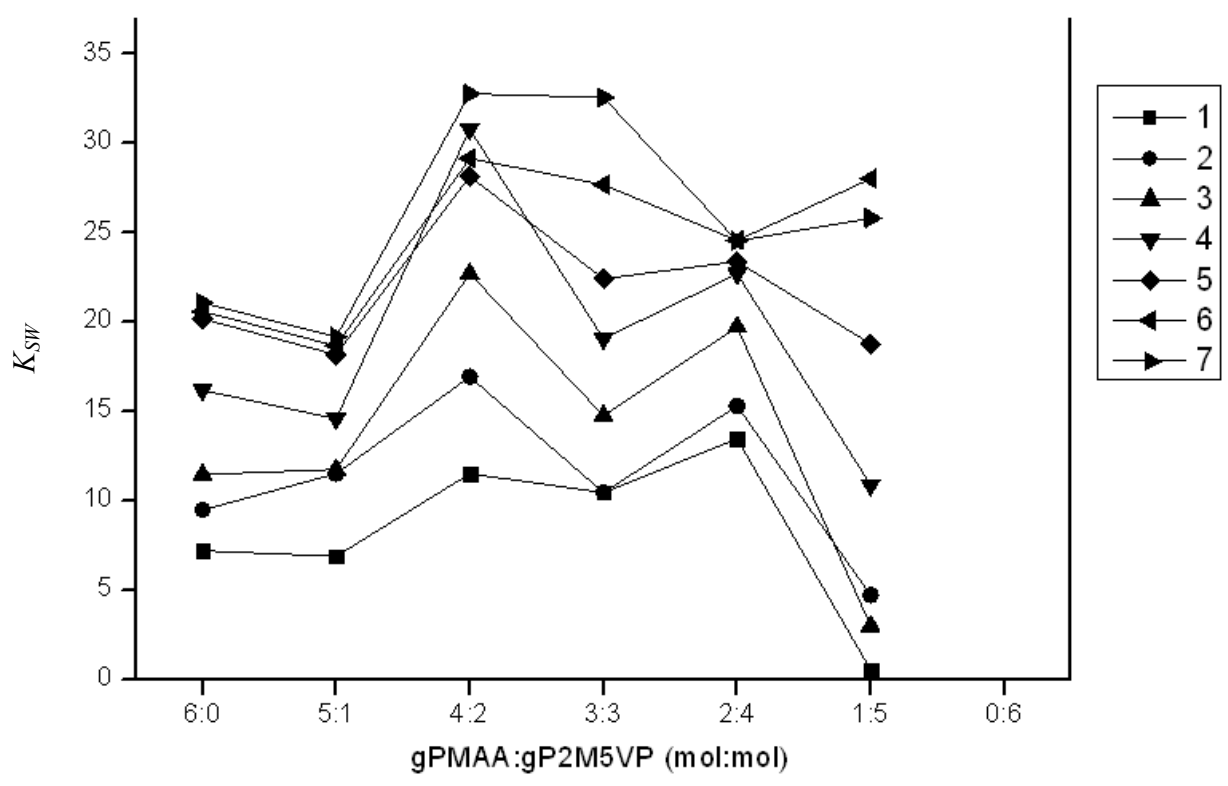

Fig. 3 Swelling coefficient dependencies of polymethacrylic acid hydrogel in presence of poly-2-methyl-5-vinylpyridine hydrogel in aqueous medium.

Curves' description: $1-20 \mathrm{~min}$; 2-40 min; 3-60 min; 4-120 min; 5-240 $\mathrm{min} ; 6$ - $480 \mathrm{~min}$; 7-1,440 min.

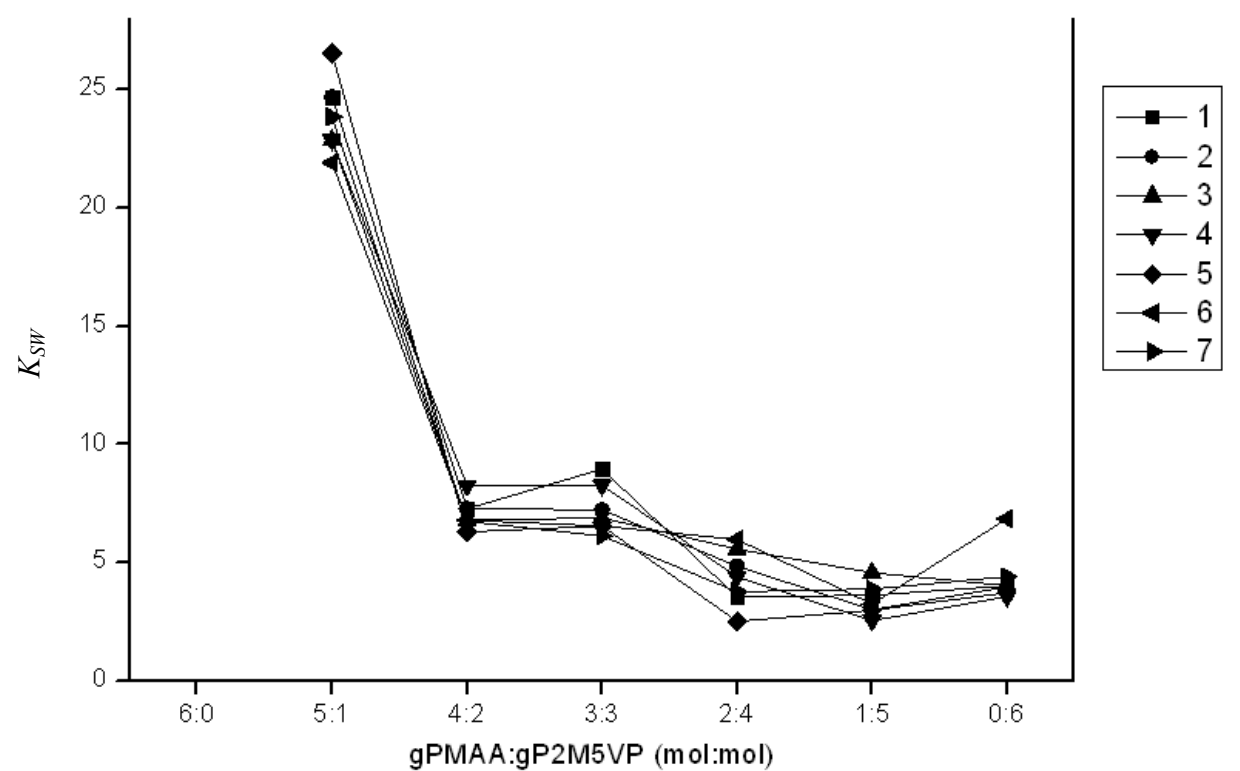

Fig. 4 Swelling coefficient dependencies of poly-2-methyl-5-vinylpyridine hydrogel in presence of polymethacrylic acid hydrogel in aqueous medium.

Curves' description: $1-20 \mathrm{~min}$; 2-40 min; 3-60 min; 4-120 min; 5-240 min; 6-480 min; 7—1,440 min.

the binding of the proton, cleaved from the carboxyl group, the by nitrogen atoms of poliobasis.

\section{Conclusions}

High electrical conductivity values indicate that for certain ratios of the two hydrogels in intergel system dissociation of carbonyl groups prevails over the association process of the proton by nitrogen heteroatoms. This may be reduction of hydrogen ion association by vinylpyridine's nitrogen atoms. The reason of this may be a conformational changes in the links of cross-site networks. At certain concentrations of charged $\mathrm{NH}^{+}$groups intramolecular crosslinks may form, such as $\geq \mathrm{N} \cdots \mathrm{H}^{+} \cdots \mathrm{N} \equiv$ which leads to the 
Remote Interaction in an Aqueous Medium

curtailment of polymer coils and reduction of proton binding.

Results, obtained in swelling coefficient study indicate that the conformational state of inter-node links is determined mainly by ionization, and not by concentration of charged groups, formed by the dissociation of functional groups.

Obtained results indicate that in an aqueous medium hydrogels of polyacrylic acid and poly-2-methyl-5-vinylpyridine interact, undergoing mutual activation on distance, and form ions that are uncomplexed and oppositely charged to ions.

\section{References}

[1] Bekturov, E. A.; Sulejmenov, I. J. Polymeric Hydrogels 1998, 237.

[2] Bekturov, E. A.; Dzhumadilov, T. K. New Approaches to the Study of Hydrogels Remote Interaction Effect. News of National Academy of Science of Republic of Kazakhstan
2009, 1, 86-87.

[3] Bekturov, E. A.; Dzhumadilov, T. K.; Korganbaeva, Z. K. Remote Effect in Polymeric Systems. Messenger of Kazakh National University after al-Farabi 2010, 3(59), 108-110.

[4] Dzhumadilov, T. K. Remote Effect of Polymeric Hydrogels Interaction in Innovative Technology. Industry of Kazakhstan 2011, 2, 70-72.

[5] Jumadilov, T.; Shaltykova, D.; Suleimenov, I. In Anomalous Ion Exchange Phenomenon, Proceedings of Austrian-Slovenian Polymer Meeting, Slovenia, Apr. 3-5, 2013.

[6] Erzhet, B.; Dzhumadilov, T. K.; Korganbaeva, Z. K. Electrochemical and Volume-Gravimetric Properties of Intergel System Based on Hydrogels of Polyacrylic Acid and Poly-4-vinylpyridine. Bulletin d'Eurotalent-Fidjip 2013, 5, 41-45.

[7] Alimbekova, B. T.; Dzhumadilov, T. K.; Korganbaeva, Z. K.; Erzhan, B.; Erzhet, B. Some Features of Interaction of Hydrogels with Low-molecular Salts. Bulletin d'Eurotalent-Fidjip 2013, 5, 28-32. 